\title{
The Effects of Anisotropy on the Nonlinear Behavior of Bridged Cracks in Long Strips
}

R. Ballarini

Case Western Reserve University

Cleveland, Ohio

and

H.A. Luo

Shanghai Jiao Tong University

Shanghai, China

February 1994

Prepared for

Lewis Research Center

Under Grant NAG3-856 


\title{
The Effects of Anisotropy on the Nonlinear Behavior of Bridged Cracks in Long Strips
}

\author{
R. Ballarini \\ Department of Civil Engineering \\ Case Western Reserve University \\ Cleveland, Ohio 44106 \\ and \\ H.A. Luo \\ Department of Engineering Mechanics \\ Shanghai Jiao Tong University \\ Shanghai 200030, China
}

\begin{abstract}
This paper presents a model which can be used to predict the twodimensional nonlinear behavior of bridged cracks in orthotropic atrips. The results obtained using a singular integral equation formulation which incorporates the anisotropy rigorously show that, although the effects of anisotropy are significant, the nondimensional quantities employed by Cox and Marshall (1991) can generate nearly universal results (R-curves, for example) for different levels of relative anisotropy. The role of composite constituent properties in the behavior of bridged cracks is clarified in this paper.
\end{abstract}

\section{Introduction}

The bridging of cracks by fibers is an important toughening mechanism in fiber reinforced brittle matrix composites (BMC). Similar characteristics are

To whom please address all correspondence. 
shared by ductile matrix composites (DMC) with weak interfaces upon cyclic loading (Evans, 1991). This fact has led to a significant amount of research in the area of stress analysis of bridged cracks. The results calculated with the models presented by Marshall, Cox and Evans (1985), Marshall and Cox. (1987), Cox and Marshall (1991), Cox (1992) and Ballarini and Muju (1991) have provided a good understanding of the fracture mechanics of bridged cracks in finite specimens. In all these models, excluding the orthotropic finite element model used by Ballarini and Muju, the anisotropy which is inherent in fiber reinforced composites was incorporated in an approximate way which may or may not provide an accurate account of its effects.

The integral equation model for a bridged crack in a finite specimen developed by Cox and co-workers can be summarized as follows. A fictitious line load $P$ per unit width of crack front was placed on one crack surface at position $x$ measured normal to the crack front. Using Castigliano's theorem the crack opening displacement (COD) $u(x)$ was determined as $u(x)=\lim _{P \rightarrow 0} \frac{\partial W}{\partial P}$, where $W$ is the elastic strain energy of the system per unit width of crack front. This energy was then written in terms of the crack length $a$, strain energy release rate $\mathcal{G}$ and stress intensity factor $K$, i.e. $W=\int_{0}^{a} \mathcal{G} d a^{\prime}=\int_{0}^{a} K^{2} / E^{\prime} d a^{\prime}$. In this equation $E^{\prime}$ is given, for an orthotropic material with a plane stress crack parallel to the principal material axis ox $x_{1}$-axis, as $1 / E^{\prime}=\sqrt{l_{11} l_{22} / 2}\left\{\sqrt{l_{22} / l_{11}}+\left(2 l_{12}+l_{68}\right) / 2 l_{11}\right\}^{1 / 2}$, where the $l_{i j}$ are compliance coefficients which enter the inverse Hooke's law, as will be explained in the next section. Cox and Marshall made the following approximation. They expressed the stress intensity factor $K$ in terms of the Green's 
function $G\left(x, a^{\prime}, w\right)$ for the isotropic material, where $w$ is a relevant characteristic length which introduces finite geometry. The representation $K=$ $2 \int_{0}^{a^{\prime}} G\left(x, a^{\prime}, w\right) \sigma(x) d x$, where $\sigma(x)$ is the traction on the crack surface, leads to the integral equation $u(x)=\left(4 / E^{\prime}\right) \int_{x}^{a} d a^{\prime}\left\{\int_{0}^{a^{\prime}} G\left(x^{\prime}, a^{\prime}, w\right) \sigma(x) d x^{\prime}\right\} G\left(x, a^{\prime}, w\right)$, which they solved numerically. This integral equation can be more directly derived using the weight function technique (Bueckner, 1970). Thus, the Green's function $G\left(x, a^{\prime}, w\right)$ can also be referred to as the Bueckner weight function. This model, by neglecting the dependency of the Green's function on the compliance coefficients, leads to the result that the COD is inversely proportional to $E^{\prime}$. Moreover, normalization of this integral equation leads to the so-called bridging length scale $a_{n}$, that is associated with a fully bridged crack in an infinitely extended material. A crack of length $a<a_{n}$ is considered short in the sense that its bridging zone is still developing. For these short fully bridged cracks the forces in the fibers are relatively small, since the crack opening displacements are small. Hence the fibers do not shield the crack tip significantly, and the stress required to propagate the matrix crack is inversely proportional to the square root of the crack length, as for the monolithic matrix. For $a \gg a_{n}$ the crack is termed long, because the bridging zone has fully developed and a steady state is reached for matrix cracking (the stress required to propagate the matrix crack is independent of crack length).

The authors have recently learned of the significant contribution to the understanding of the role of material orthotropy in fracture specimens for composites made by Suo et al. (1991). They found a spatial rescaling that could 
be used to reduce boundary value problems (BVP) involving orthotropic materials to equivalent problems in materials with cubic symmetry. Under certain conditions the cubic-symmetric materials may be approximated by isotropic materials. Therefore the rescaling technique can be used in conjunction with existing isotropic material solutions to construct approximate orthotropic material solutions. These spatial rescaling relations, which will be discussed subsequently, were used by Bao et al. (1992) in their analysis of commonly used fracture specimens to investigate the interplay between material anisotropy and finite geometry. In their finite element method analysis the role of orthotropy in fiber bridging phenomena was not considered.

The work presented in this paper was initiated to investigate the effects of relative anisotropy on the behavior of bridged cracks in long strips so that the assumption used in the aforementioned analyses of bridged cracks could be assessed. Moreover, an efficient and highly accurate analytical model based on dislocation theory was sought that will facilitate the analysis of experiments conducted on beam specimens. The results obtained using a singular integral equation formulation which incorporates rigorously the effects of orthotropy show that the weight function $G\left(x^{\prime}, a^{\prime}, w\right)$ depends not only on $E^{\prime}$ but also other stiffness coefficients. However, the numerical calculations presented in this paper indicate that for the unbridged cracks in different orthotropic strips the CODs are indeed approximately inversely proportional to their values of $E^{\gamma}$. A wide range of computations for the nonlinear behavior of bridged cracks also supports to adopt simple approximate weight functions which are derived for the corresponding isotropic specimen. These results 
generalize the conclusions made by Bao et al. to fiber-bridged cracks in long strips. Evidently, this conclusion will facilitate the analysis of R-curves for bridged cracks in orthotropic beam specimens.

\section{Formulation}

Consider an infinite orthotropic plate whose principal material axes coincide with the coordinate axes. A unit dislocation with Burgers vector $b_{x}=1$ is embedded at $P\left(0, y_{0}\right)$. The stresses in the plate are (Milne-Thomson, 1960):

$$
\begin{aligned}
& \sigma_{x z}^{(1)}=\frac{1}{2} \operatorname{Re}\left[\frac{A_{1} \lambda_{1}^{2}}{z_{1}}+\frac{A_{2} \lambda_{2}^{2}}{z_{2}}\right], \quad \sigma_{y y}^{(1)}=\frac{1}{2} \operatorname{Re}\left[\frac{A_{1}}{z_{1}}+\frac{A_{2}}{z_{2}}\right], \\
& \sigma_{x y}^{(1)}=-\frac{1}{2} \operatorname{Re}\left[\frac{A_{1} \lambda_{1}}{z_{1}}+\frac{A_{2} \lambda_{2}}{z_{2}}\right],
\end{aligned}
$$

where

$$
z_{1}=x+\lambda_{1}\left(y-y_{0}\right), \quad z_{2}=x+\lambda_{2}\left(y-y_{0}\right)
$$

$\lambda_{1}$ and $\lambda_{2}$ are two roots of the characteristic equation

$$
l_{12} \lambda^{4}+\left(2 l_{12}+l_{68}\right) \lambda^{2}+l_{22}=0
$$

with $\operatorname{Im}\left(\lambda_{1}\right) \geq \operatorname{Im}\left(\lambda_{2}\right)>0, \lambda_{3}=-\lambda_{1}, \lambda_{4}=-\lambda_{2}$. The $l_{i j}$ are the anisotropic compliance coefficients which enter the inverse Hooke's law:

$$
\left(\begin{array}{c}
\epsilon_{z x} \\
\epsilon_{y y} \\
2 \epsilon_{x y}
\end{array}\right)=\left(\begin{array}{ccc}
l_{11} & l_{12} & 0 \\
l_{12} & l_{22} & 0 \\
0 & 0 & l_{66}
\end{array}\right)\left(\begin{array}{c}
\sigma_{z x} \\
\sigma_{y y} \\
\sigma_{z y}
\end{array}\right) .
$$


In equation (1), the constants $A_{1}$ and $A_{2}$ are determined by

$$
\left\{\begin{array}{l}
p_{1} A_{1}+p_{2} A_{2}-\bar{q}_{1} \bar{A}_{1}-\bar{q}_{2} \bar{A}_{2}=2 / \pi i \\
\delta_{1} A_{1}+\delta_{2} A_{2}-\bar{\gamma}_{1} \bar{A}_{1}-\bar{\gamma}_{2} \bar{A}_{2}=0
\end{array}\right.
$$

where

$$
\begin{aligned}
& p_{1}=l_{12}+l_{11} \lambda_{1}^{2}+i\left(l_{22}+l_{12} \lambda_{1}^{2}\right) / \lambda_{1}, \\
& p_{2}=l_{12}+l_{11} \lambda_{2}^{2}+i\left(l_{22}+l_{12} \lambda_{2}^{2}\right) / \lambda_{2}, \\
& \bar{q}_{1}=l_{12}+l_{11} \bar{\lambda}_{1}^{2}+i\left(l_{22}+l_{12} \bar{\lambda}_{1}^{2}\right) / \bar{\lambda}_{1}, \\
& \bar{q}_{2}=l_{12}+l_{11} \bar{\lambda}_{2}^{2}+i\left(l_{22}+l_{12} \bar{\lambda}_{2}^{2}\right) / \bar{\lambda}_{2}, \\
& \dot{\delta}_{1}=\left(1+i \lambda_{1}\right) / 2, \quad \delta_{2}=\left(1+i \lambda_{2}\right) / 2, \\
& \gamma_{1}=\left(1-i \lambda_{1}\right) / 2, \quad \gamma_{2}=\left(1-i \lambda_{2}\right) / 2 .
\end{aligned}
$$

The first equation in (5) represents the necessary displacement jump condition, while the second represents zero net force on the dislocation.

- When the dislocation is located in an orthotropic strip (Figure 1), an additional solution must be superposed to satisfy the traction free boundary conditions on the surfaces $y=0$ and $y=h$. The stresses from the additional solution, denoted by superscript (2), must satisfy

$$
\begin{array}{ll}
\sigma_{w y}^{(2)}(x, 0)=-\sigma_{w y}^{(1)}(x, 0), & \sigma_{x y}^{(2)}(x, 0)=-\sigma_{x y}^{(1)}(x, 0), \\
\sigma_{w y}^{(2)}(x, h)=-\sigma_{w y}^{(1)}(x, h), & \sigma_{z y}^{(2)}(x, h)=-\sigma_{x y}^{(1)}(x, h) .
\end{array}
$$

This part of the solution can be determined using Fourier transformation techniques. Its displacement components are expressed as

$$
u^{(2)}(x, y)=\frac{2}{\pi} \int_{0}^{\infty} U(\xi, y) \sin \xi x d x
$$




$$
v^{(2)}(x, y)=\frac{2}{\pi} \int_{0}^{\infty} V(\xi, y) \cos \xi x d x
$$

with

$$
\begin{aligned}
& U(\xi, y)=\sum_{j=1}^{4} B_{j}(\xi) \exp \left(s_{j} \xi y\right) \\
& V(\xi, y)=\sum_{j=1}^{4} d_{j} B_{j}(\xi) \exp \left(s_{j} \xi y\right)
\end{aligned}
$$

where

$$
d_{j}=\left[\beta_{2} s_{j}^{s}-\left(\beta_{1} \beta_{2}-\beta_{3}^{2}\right) s_{j}\right] / \beta_{3}
$$

and the $s_{j}(j=1,2, \ldots, 4)$ are the roots of the equation

$$
s^{4}+\frac{\beta_{3}^{2}-\beta_{1} \beta_{2}-1}{\beta_{2}} s^{2}+\frac{\beta_{1}}{\beta_{2}}=0
$$

In (10) and (11)

$$
\beta_{1}=b_{11} / G_{12}, \quad \beta_{2}=b_{22} / G_{12}, \quad \beta_{3}=1+b_{12} / G_{12},
$$

where the $b_{i j}$ and $G_{12}$ are the stiffness coefficients of the material:

$$
\left(\begin{array}{c}
\sigma_{x x} \\
\sigma_{y y} \\
\sigma_{x y}
\end{array}\right)=\left(\begin{array}{ccc}
b_{11} & b_{12} & 0 \\
b_{12} & b_{22} & 0 \\
0 & 0 & G_{12}
\end{array}\right)\left(\begin{array}{c}
\epsilon_{x z} \\
\epsilon_{y y} \\
2 \epsilon_{x y}
\end{array}\right) .
$$

Using condition ( 7$)$, the coefficients $B_{j}(\xi)(j=1,2, \ldots, 4)$ can be determined by solving the set of linear equations given in the Appendix.

Along the $y$-axis the resultant stress $\sigma_{x z}$ in the strip is

$$
\sigma_{x x}(0, y)=\frac{\kappa}{y_{0}-y}+G\left(y, y_{0}\right)
$$


with

$$
\begin{aligned}
& G\left(y, y_{0}\right)=\frac{2 b_{11}}{\pi} \sum_{j=1}^{4} \int_{0}^{\infty} B_{j}(\xi) \xi e^{s_{j} \xi \eta} d \xi+\frac{2 b_{12}}{\pi} \sum_{j=1}^{4} d_{j} s_{j} \int_{0}^{\infty} B_{j}(\xi) \xi e^{u_{j} \xi y} d \xi \\
& \kappa=-\operatorname{Re}\left(A_{1} \lambda_{1}+A_{2} \lambda_{2}\right) / 2 .
\end{aligned}
$$

The stiffness parameter $\kappa$ plays a crucial role in defining the level of anisotropy of a given material system. After comparing it with the relevant quantities $E^{\prime}$ and $A$ which are adopted by Cox and Marshall (1991) and Budiansky and Amazigo (1989), respectively, it is found that

$$
\kappa=\frac{E^{\prime}}{4 \pi}=\frac{A E_{11}}{4 \pi\left(1-\nu_{m}^{2}\right)}
$$

where $\nu_{m}$ is the matrix Poisson's ratio, $E_{11}$ the longitudinal composite Young's modulus. Because the crack is now perpendicular to the $x$-axis, in equation (16)

$$
\frac{1}{E^{\prime}}=\left(\frac{l_{11} l_{22}}{2}\right)^{1 / 2}\left[\left(\frac{l_{11}}{l_{22}}\right)^{1 / 2}+\frac{2 l_{12}+l_{66}}{2 l_{22}}\right]^{1 / 2}
$$

This quantity was first introduced by Sih (1968).

With (17) the relative in-plane orthotropy is specified through three ratios: $l_{22} / l_{11}, l_{12} / l_{11}$ and $l_{66} / l_{11}$. Suo $(1990 \mathrm{a}, \mathrm{b})$ introduced two parameters

$$
\lambda=\frac{l_{11}}{l_{22}}, \quad \rho=\frac{2 l_{12}+l_{66}}{2 \sqrt{l_{11} l_{22}}}
$$

as the only two parameters needed to quantify the level of orthotropy. $\lambda=$ $\rho=1$ for isotropic solids and $\lambda=1$ for solids with cubic symmetry. Using Lekhnitskii's formalism (1981) he showed that in simply-connected sheets 
with traction prescribed on the boundary the governing equation for the Airy stress function can be written as

$$
\frac{\partial^{4} U}{\partial x^{4}}+2 \rho \sqrt{\lambda} \frac{\partial^{4} U}{\partial x^{2} \partial y^{2}}+\lambda \frac{\partial^{4} U}{\partial y^{4}}=0
$$

This suggests that the stresses depend on material properties only through $\rho$ and $\lambda$. Furthermore, by rescaling the $x$-axis by $\xi=\lambda^{1 / 4} x$ the $\lambda$ dependence can be extracted explicitly, so that in the transformed plane the stresses depend only on $\rho$, i.e.,

$$
\frac{\partial^{4} U}{\partial \xi^{4}}+2 \rho \frac{\partial^{4} U}{\partial \xi^{2} \partial y^{2}}+\frac{\partial^{4} U}{\partial y^{4}}=0
$$

Obviously, solution to BVP for the class of materials obeying $\rho=1(\lambda \neq$ 1) can be constructed from the solution to the corresponding BVP for the isotropic material. It can be shown that

$$
\frac{1}{E^{\prime}}=I_{22} \sqrt{\frac{\lambda^{3 / 2}(1+\rho)}{2}}
$$

for $\mathrm{a}$ crack in the $y$-direction. The parameters $\lambda$ and $\rho$ will be considered in subsequent calculations.

Consider a cracked strip shown in Figure 1 and introduce parameters $t$ and $\tau$ such that $y=b+a t / 2$ and $y_{0}=b+a \tau / 2$. The discrete dislocation is replaced with a distribution of dislocations

$$
B_{a}(\tau) \equiv-\frac{2}{a} \frac{\partial}{\partial \tau}\left[u\left(0^{+}, \tau\right)-v\left(0^{-}, \tau\right)\right]
$$

This representation enables one to write the following singular integral equation for the traction boundary condition along the crack, with expression 
(14) being the appropriate kernel:

$$
\int_{-1}^{1} \frac{B_{x}(\tau)}{\tau-t} d \tau+\int_{-1}^{1} K(t, \tau) B_{z}(\tau) d \tau=-q(t)
$$

where $K(t, \tau)$ is the regular part of the kernel

$$
K(t, \tau)=\frac{a}{2 \kappa} G(b+a t / 2, b+a \tau / 2),
$$

and the loading term is given by

$$
q(t)=\frac{1}{\kappa}\left[\sigma_{A}(t)-p(t)\right]
$$

Here $\sigma_{A}(t)$ is the stress caused by the applied load in the crack-free strip and $p(t)$ is the closing stress of the bridging fibers. In general, $p(t)=p(u(t))$ where $u(t)$ is the crack opening displacement at $t$. For internal cracks, the crack closure condition

$$
\int_{-1}^{1} B_{x}(\tau) d \tau=0
$$

should be supplemented to equation (23). The square root stress singularity at crack tips is modeled by expressing $B_{x}(\tau)$ as

$$
B_{n}(\tau)=\phi(\tau) / \sqrt{1-\tau^{2}}
$$

for internal cracks and

$$
B_{*}(\tau)=\phi(\tau) / \sqrt{1-\tau}
$$

for edge cracks, where $\phi(\tau)$ is a regular function.

It is important to note that unlike the isotropic case, where the kernel of the singular integral equation is independent of the elastic constants, the 
kernel which appears in (23) depends on the ratios $E_{22} / E_{11}, G_{12} / E_{11}$ and $\nu_{12}$ for the plane stress case and $E_{22} / E_{11}, E_{33} / E_{11}, G_{12} / E_{11}, \nu_{12}, \nu_{1 s}$ and $\nu_{29}$ for the plane deformation case. Nevertheless, from a viewpoint of the orthotropy rescaling, in the transformed $\xi y$-plane the stress field for a unit dislocation $b_{x}=1$ embedded in a long strip is determined by governing equation (20). The corresponding boundary conditions are

$$
\frac{\partial^{2} U}{\partial \xi^{2}}=0, \quad \frac{\partial^{2} U}{\partial \xi \partial y}=0
$$

at $y=0$ and $y=h$. On the dislocation, the zero net force condition and the displacement jump condition are (Bao et al., 1992)

$$
\left[\frac{\partial U}{\partial y}\right]_{L}=0, \quad\left[\frac{\partial U}{\partial \xi}\right]_{L}=0
$$

and

$$
\left[\frac{\partial \chi}{\partial y}\right]_{L}=4 \pi \kappa \sqrt{\frac{1+\rho}{2}}, \quad\left[\frac{\partial \chi}{\partial \xi}\right]_{L}=0
$$

respectively, where the symbol []$_{L}$ denotes the increment received on passing once round a closed curve enclosing the dislocation and $\chi$ is the auxiliary function defined by

$$
\frac{\partial^{2} \chi}{\partial \xi \partial y}=\frac{\partial^{2} U}{\partial \xi^{2}}+\frac{\partial^{2} U}{\partial y^{2}}, \quad 2(1-\rho) \frac{\partial^{2} U}{\partial \xi \partial y}=\frac{\partial^{2} \chi}{\partial \xi^{2}}+\frac{\partial^{2} \chi}{\partial y^{2}}
$$

Therefore, the kernel of equation (23), which is equal to $(1 / \kappa)\left(\partial^{2} U / \partial y^{2}\right)$, depends on elastic constants only through the nondimensional parameters $\lambda$ and $\rho$. Moreover, because in Figure 1 there is no characteristic length in the $x$-direction the kernel actually is a function of $\rho$ only. 
The above integral equations are solved numerically. By approximating $\phi(t)$ as piecewise quadratic polynomials, equations (23) and (26) are reduced to:

$$
\sum_{j=1}^{n} M_{i j} \phi\left(\tau_{j}\right)=-\frac{1}{\kappa}\left[\sigma_{A}\left(t_{i}\right)-p\left(u\left(t_{i}\right)\right)\right.
$$

where $\tau_{j}$ is the integral point, $t_{i}$ is the collocation point, and the matrix element $M_{i j}$ consists of the weights given in Gerasoulis (1982). For edge cracks $i=1,2, \ldots, n$, and for internal cracks $i=1,2, \ldots, n-1$ with the nth equation

$$
\sum_{j=1}^{n} M_{n j} \phi\left(\tau_{j}\right)=0
$$

coming from (26). In the following discussion, we only consider edge cracked and center cracked strips.

Equation (33) represents a set of nonlinear equations for the unknown dislocation distribution. A more efficient iteration procedure is obtained by deriving a compliance matrix, as outlined in Ballarini (1986).

From (33) and (34),

$$
\phi\left(\tau_{j}\right)=-\frac{1}{\kappa} \sum_{k=1}^{n} N_{j k}\left[\sigma_{A}\left(\tau_{k}\right)-p\left(u\left(\tau_{k}\right)\right)\right] \quad(j=1,2, \ldots, n)
$$

where $N=M^{-1} T$ with $M^{-1}$ denoting the inverse of matrix $M$, and $T$ is an $n \times n$ matrix which transforms the coordinates from the integral points to the collocation points. Following Ballarini (1986), by substituting (35) into (28) and integrating $B_{x}(\tau)$ from $\tau_{j}$ to 1 , equation (35) is cast in the form:

$$
u\left(\tau_{j}\right)=\frac{a}{k} \sum_{k=1}^{n-1} C_{j k}\left[\sigma_{A}\left(\tau_{k}\right)-p\left(u\left(\tau_{k}\right)\right)\right] \quad(j=1,2, \ldots, n-1)
$$


where the matrix $C$ is known as the compliance matrix of the crack which relates the stress on the crack face with the crack opening displacement. Thus, a system of nonlinear algebraic equations for crack opening displacement $u\left(\tau_{j}\right)$ is obtained.

The total stress intensity factor which includes the fiber shielding effects is

$$
\begin{aligned}
K_{T} & =-\eta \kappa \pi \sqrt{\pi a} \phi(1) \\
& =\eta \pi \sqrt{\pi a} \sum_{k=1}^{n} N_{n k}\left[\sigma_{A}\left(\tau_{k}\right)-p\left(u\left(\tau_{k}\right)\right)\right]
\end{aligned}
$$

while the so-called shielding stress intensity factor is given by

$$
K_{F}=\eta \pi \sqrt{\pi a} \sum_{k=1}^{n} N_{n h p}\left(u\left(\tau_{k}\right)\right)
$$

Here $\eta=1$ for edge cracks and $\eta=1 / \sqrt{2}$ for center cracks, respectively. It is obvious that $K_{T}=K_{A}-K_{F}$ where $K_{A}$ is the stress intensity factor due to applied load only.

\section{Results and Discussion}

\section{Zero flber bridging}

To check the numerical procedure calculations were first performed for the case of zero fiber bridging stress. The computed stress intensity factors and crack mouth opening displacement (CMOD) for uniform tension and bending, for various values of relative orthotropy and crack length, are practically the same as those reported by Delale and Erdogan (1977) and Kaya and Erdogan (1980), and are not presented here. Selected results for plane 
stress edge cracks under uniform tension are presented in Table 1 and 2 to highlight the significance of material anisotropy.

Table 1 and 2 show the normalized stress intensity factor $K / \sigma_{\infty} \sqrt{\pi a / 2}$ and normalized crack mouth opening displacement $[u] E^{\prime} / 2 \sigma_{\infty} h$, respectively, as functions of the orthotropy rescaling factors $\lambda$ and $\rho$. It is observed that for a given crack length, both the quantities are independent of $\lambda$ and are weak functions of $\rho$. This is because the kernel of equation (23) is a function of $\rho$ only. As a result, the parameter $\rho$ will slightly change the proportionality of $[u]$ to $1 / E^{\prime}$. Such effects also exist on the relation between bridging force and relative anisotropy.

The normalized COD profile for a plane stress edge crack of length equal to one half the depth of the beam is plotted in Figure 2 as functions of relative orthotropy which is obtained by keeping the values of $E_{22}=E_{93}$, $G_{12}=E_{22} / 2\left(1+\nu_{12}\right), \nu_{12}=\nu_{13}=\nu_{29}=0.3$ unchanged while varying the value of $E_{11}$ only. For the cases $E_{11} / E_{22}=1,2,5$ and $10, E^{\prime} / E_{22}=1,1.47,2.43$ and $3.55, \lambda=1,0.5,0.2$ and 0.1 , and $\rho=1,1.63,2.77$ and 4.02 , respectively. It is seen that, though the material anisotropy has significant influence on the COD, the relation between $[u] E^{y} / 2 \sigma_{\infty} t h$ and $y / a$ is almost independent of the level of relative orthotropy. The largest discrepancy occurs at the crack mouth, but even for $E_{11} / E_{22}=10$ it is less than $8 \%$ compared with the isotropic case.

\section{Initially fully bridged cracks}

Results are calculated next for bridging fibers whose strength satisfies a 
two parameter Weibull distribution and whose sliding is resisted by a constant frictional stress $\tau$. The closing stress can be approximated as (Cox and Marshall, 1991)

$$
p(u)=f \Sigma U^{1 / 2} \exp \left(-U^{(m+1) / 2}\right)
$$

where $f$ is the volume fraction of fibers, $m$ is the Weibull modulus and $U$ is the normalized COD:

$$
\begin{aligned}
& U=u / u_{n}, \quad u_{n}=\frac{\Sigma^{2} R(1-f) E_{m}}{4 \tau E_{f} E_{11}} \\
& \Sigma=\langle s\rangle / \Gamma\left(\frac{m+1}{m+2}\right)
\end{aligned}
$$

with $\langle s\rangle$ the average fiber strength, $\Gamma$ the gamma function, $R$ the fiber radius, and $E_{\mathrm{m}}, E_{f}$ and $E_{11}$ the moduli of the matrix, fiber and composite in the fiber direction.

The effects of anisotropy are demonstrated through the specific example of two plane strain orthotropic strips: strip 1, nearly isotropic with bulk properties $E_{11}=279.6 \mathrm{GPa}, E_{22}=E_{33}=253.9 \mathrm{GPa}, G_{12}=97.66 \mathrm{GPa}$ and $\nu_{12}=\nu_{13}=\nu_{23}=0.3$, and strip 2, with all properties equal to those of strip 1 , except $E_{11}=1398 \mathrm{GPa}$. Their rescaling parameters are $\lambda=0.9163$ and $\rho=1.086$ for strip 1 and $\lambda=0.1963$ and $\rho=3.048$ for strip 2 .

For each strip three different normalized crack lengths were considered: $A=3,5,7$ for uniform tension loading where

$$
A=a / a_{n}, \quad a_{n}=\frac{\pi \Sigma R(1-f) E_{m} E^{y}}{16 \tau f E_{f} E_{11}}
$$


The dimensionless strip width is $H=h / a_{n}=10$.

In each analysis the crack length is held fixed as the loading is increased. By controlling the length of the bridged crack results are obtained which highlight the transition from stable to unstable behavior of the crack. The resulting physical parameters are presented in Figures 3-8. These include the square root of the normalized crack mouth opening displacement $\sqrt{U_{0}}=$ $\sqrt{u(-1) / u_{n}}$, square roots of the normalized total and shielding stress intensity factors $\sqrt{K_{T} / K_{0}}$ and $\sqrt{K_{F} / K_{0}}$ as functions of normalized loading parameter $\sqrt{S_{e}}$, where

$$
K_{0}=2 f \Sigma \sqrt{2 a_{n} / 3 \pi}
$$

$S_{\varepsilon}=\sigma_{\infty} / f \Sigma$ for uniform tension under stress $\sigma_{\infty}$.

It should be noted that the nondimensional variables used in equations (39)-(42) were defined by Cox and Marshall (1991). They are adopted in the present analysis to drive home the point whether this set of nondimensional parameters are sufficient to predict the behavior of bridged cracks in composite materials which possess different levels of anisotropy.

Notice that for a bridged Griffith crack $(b=h / 2, a / h \rightarrow 0)$, the effects of free surfaces disappear, and the Fourier transform kernel $K(t, \tau)$ vanishes. Consequently, the compliance matrix $\mathbf{C}$ in equation (36) is independent of elastic moduli. As illustrated by Figures 3-5, where the Weibull modulus $m=1$, the relations between $U_{0}$ and $S_{a}, K_{T} / K_{0}$ and $S_{a}$ and $K_{F} / K_{0}$ and $S_{\alpha}$ are universal for all orthotropic strips with common values of $A$.

The stability of the bridged cracks can be viewed either through the $\sqrt{U_{0}}$ 
versus $\sqrt{S_{a}}, \sqrt{K_{T} / K_{0}}$ versus $\sqrt{S_{a}}$ or $\sqrt{K_{F} / K_{0}}$ versus $\sqrt{S_{a}}$ plots. Figure 3 shows that for relatively short cracks an increase in losd is needed to increase the crack mouth opening displacement (CMOD). For long cracks, on the other hand, as the fibers start pulling out the CMOD increases even if the load is decreased. In other words, the instability is indicated by a discontinuity in the CMOD.

Consider next the stress intensity factors. Figure 5 shows that for the stable short cracks, the shielding produced by the fibers varies stably, while for long cracks there is a sharp reduction in shielding as the fibers pull out. This sharp reduction corresponds to the discontinuous increase in total stress intensity factor in Figure 4.

Of interest is how the normalized quantities $A$ and $H$ work when the orthotropic strips have finite geometry configurations. Figures 6-8 show the curves $\sqrt{U_{0}}$ versus $\sqrt{S_{a}}, K_{T} / K_{0}$ versus $\sqrt{S_{a}}$ and $K_{F} / K_{0}$ versus $\sqrt{S_{a}}$ for bridged edge cracks with $m=0$ under uniform tension. From these figures it is seen that even in finite geometries the above normalized quantities can still yield nearly universal results, regardless of the level of anisotropy. Although they are not presented here, the results for bending loading and for center cracked strips showed similar trends.

The above results suggest that the regular kernel $K(t, \tau)$ in equation (23) can be expressed as $K^{0}(t, \tau)[1+Y(p, a / h, b / h)]$ where $K^{0}(t, \tau)$ is the regular kernel of the corresponding isotropic cracked strip and $Y(\rho, a / h, b / h)$ is a higher order perturbation term which is a function of $\rho, a / h$ and $b / h$ with 
$Y(1, a / h, b / h)=0$ and $Y(p, 0,0.5)=-1$. To obtain the detailed form of $Y(\rho, a / h, b / h)$ further numerical investigation is needed and is not discussed in this paper.

Here it should be emphasized that although in all the above calculations only the bulk moduli enter (4) and (13), for real fiber-reinforced composites the bulk moduli are related to the constituent properties. There are many procedures published in the literature to perform this homogenization process (Mura, 1987).

From the foregoing analysis it is seen that the length parameter $a_{n}$ is a key parameter to define all relevant normalized quantities such as the normalized crack length $A$, normalized strip width $H$, normalized stress intensity factors $K_{T} / K_{0}$ and $K_{F} / K_{0}$. Then, what role is played by the constituent properties in determining the value of $a_{n}$ ? To this end, the parameter $a_{n}$ is written as

$$
a_{n}=\frac{\pi}{16} \frac{\boldsymbol{\Sigma} R}{\tau} \alpha
$$

where

$$
\alpha=\frac{(1-f) E_{m} E^{\prime}}{f E_{f} E_{11}}
$$

which comprehensively reflects the infiuence of the constituent stiffiness and the fiber volume fraction on $a_{n}$.

Consider the following composite whose fibers are aligned along the $x$-axis with $E_{f} / E_{m}=10, \nu_{f}=0.3$ and $\nu_{m}=0.35$ where $E_{f}$ and $E_{m}$ are Young's moduli of the fiber and matrix, respectively, and $\nu_{f}$ and $\nu_{m}$ are their Poisson's ratios. The well-known Mori-Tanakn method (Luo and Weng, 1989) was used 
to determine the five effective elastic constants of the composite strip. Figure 9 shows $\alpha, E^{y} / E_{m}$ and $E_{11} / E_{m}$ as functions of $f$ for plane strain. Figure 10 plots $\alpha$ versus $f$ for several values of $E_{f} / E_{m}$. Figure 11 shows the variation of $\alpha, E^{\prime} / E_{m}$ and $E_{11} / E_{m}$ with respect to $E_{f} / E_{m}$ for $f=0.4$. It is observed that when the volume fraction is low, $\alpha$ sharply decreases with an increase in $f$, while beyond this range $\alpha$ changes slowly. Besides, $\alpha$ decreases with increasing $E_{f} / E_{\mathrm{m}}$. From the above observations it is clear that, since large values of $A$ in general correspond to less stable bridged cracks, then for $a$ given length of bridged crack increasing the stiffness ratio $E_{f} / E_{m}$ or volume fraction $f$ will reduce the stability of the bridged crack. However, this can be compensated by increasing the fiber strength $\Sigma$, fiber radius $R$ and decreasing the interfacial frictional friction stress $\tau$.

\section{Concluding Remarks}

A singular integral equation formulation has been presented for the analysis of bridged cracks in orthotropic strips. The exact solutions given in this paper show that in terms of the normalized parameters introduced by Cox and Marshall (1991) the nonlinear behavior of bridged cracks can be depicted in a nearly universal form for materials possessing different levels of anisotropy. The increase of the ratio of stiffness $E_{f} / E_{m}$ and volume fraction $f$ in general reduces the stability of the bridged cracks, which can be remedied by increasing the fiber strength and fiber radius and decreasing the interfacial frictional friction stress. 


\section{Acknowledgement}

H.A.L. acknowledges support from the National Natural Science Foundation of China and the National Lab of MMC at Shanghai Jiao Tong University; R.B. acknowledges support from NASA-Lewis (Grant NAG3-856) and ONR (contract N0013-86-K-0773).

\section{References}

Ballarini, R. (1986). Compliance matrices for cracked bodies. Int. J. Fracture 31, R63-R66.

Ballarini, R. and Muju, S. (1993). Stability analysis of bridged cracks in brittle matrix composites. J. Engng. Gas Turbines Power 115, 127-138.

Bao, G., Ho, S., Suo, Z. and Fan B. (1992). The role of material orthotropy in fracture specimens for composites. Int. J. Solids Struct. 29, 1105-1116.

Budiansky, B. and Amazigo, J.C. (1989). Toughening by aligned, frictionally constrained fibers. J. Mech. Phys. Solids 37, 93-109.

Bueckner, H.F. (1970). A novel principle for the computation of stress intensity factors. Z. Angewandte Mathemat. Mechan. 50, 529-546.

Cox, B.N. and Marshall, D.B. (1991). Stable and unstable solutions for bridged cracks in various specimens. Acta Metall. 39, 579-589.

Cox, B.N. (1991). Extrinsic factors in the mechanics of bridged cracks. Acta Metall. 39, 1189-1201.

Delale, F. and Erdogan, F. (1977). The problem of internal and edge cracks in an orthotropic strip. J. Appl. Mech. 44, 237-242.

Evans, A.G. (1991). The mechanical properties of reinforced ceramic, metal and intermetallic matrix composites. Mater. Sci. Engng. A143, 63-76.

Gerasoulis, A. (1982). The use of piecewise quadratic polynominals for the solution of singular integral equations of Cauchy type. Comput. Math. Appl. 8, 15-22. 
Kaya, A.C. and Erdogan, F. (1980). Stress intensity factors and COD in an orthotropic strip. Int. J. Fracture 16, 171-190.

Lekhnitskii, S.G. (1981). Theory of Elasticity of an Anisotropic Body, Mir Publishers, Moscow.

Luo, H.A. and Weng, G.J. (1989). On Eshelby's S-tensor in a three-phase cylindrically concentric solid, and the elastic moduli of fiber-reinforced composites. Mech. Mater. 8, 77-88.

Marshall, D.B. and Cox, B.N. (1987). Tensile fracture of brittle matrix composites: influence of fiber strength. Acta Metall. 35, 2607-2619.

Marshall, D.B., Cox, B.N. and Evans, A.G. (1985). The mechanics of matrix cracking in brittle matrix fiber composites. Acta Metall. 33, 2013-2021.

Milne-Thomson, L.M. (1960). Plane Elastic Systems, Springer-Verlag, Berlin.

Mura, T. (1987). Micromechanics of Defects in Solids, Martinus Nijhoff, Dordrecht.

Sih, G.C. and Liebowitz, H. (1968). Mathematical theories of brittle fracture. Fracture, II, 67-190 (edited by H. Liebowitz), Academic, New York.

Suo, Z. (1990a). Delamination specimens for orthotropic materials. J. Appl. Mech. $57,627-634$.

Suo, Z. (1990b). Singularities, interfaces and cracks in dissimilar anisotropic media. Proc. R. Soc. Lond. A427, 331-358.

Suo, Z., Bao, G., Fan, B. and Wang, T.C. (1991). Orthotropy rescaling and implications for fracture in composites. Int. J. Solids Struct. 28, 235-248. 


\section{Appendix}

In equation $(9), B_{j}(\xi)$ is determined by

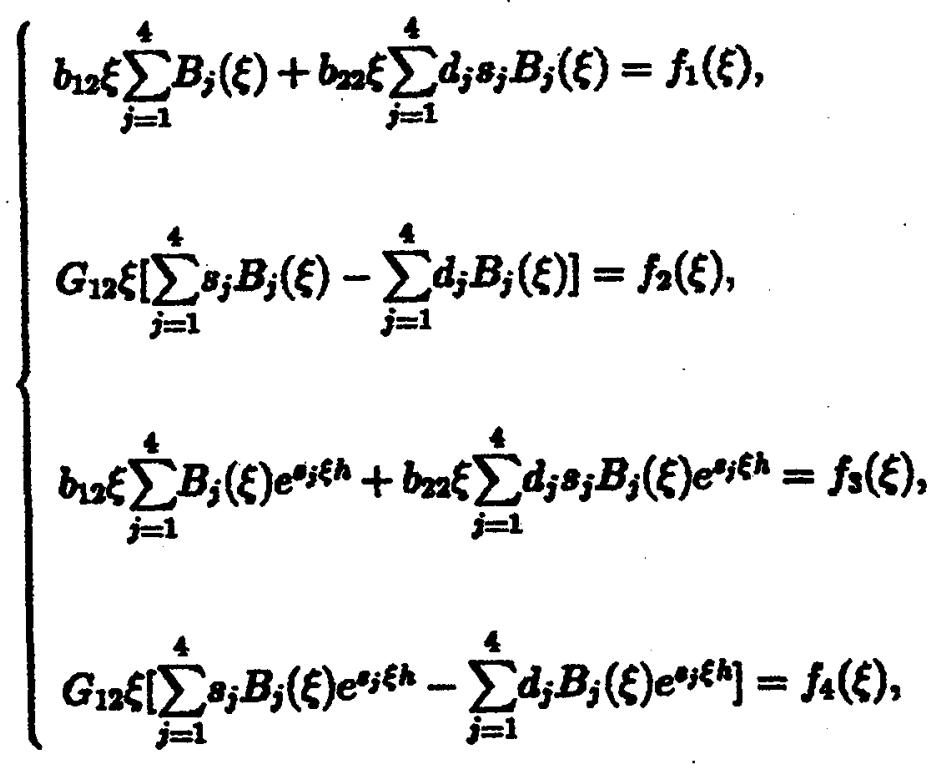

where

$$
\begin{aligned}
& f_{1}(\xi)=-\frac{\pi i}{4}\left[A_{1} e^{i \lambda_{2} \omega \xi}+A_{2} e^{i \lambda_{2} \cos \xi}\right] \\
& f_{2}(\xi)=\frac{\pi}{4}\left[A_{1} \lambda_{1} e^{i \lambda_{2} \operatorname{lno} \xi}+A_{2} \lambda_{2} e^{i \lambda_{2} 00 \xi}\right] \\
& f(\xi)=-\frac{\pi i}{4}\left[\bar{A}_{1} e^{-i \lambda_{1}\left(h_{-}-\infty\right) \xi}+\bar{A}_{2} e^{-i \lambda_{2}(h-\infty) \mathcal{E}}\right], \\
& f_{1}(\xi)=\frac{\pi}{4}\left[\bar{A}_{1} \bar{\lambda}_{1} e^{i \bar{\lambda}_{1}(h-\infty) x}+\bar{A}_{2} \bar{\lambda}_{2} e^{i \lambda_{2}(h-\infty) k}\right]
\end{aligned}
$$




\section{Table Captions}

Table 1 Normalized stress intensity factor vs. orthotropy rescaling parameters $\lambda$ and $\rho$ for zero fiber bridged edge cracks.

Table 2 Normalized CMOD vs. orthotropy rescaling parameters $\lambda$ and $\rho$ for zero fiber bridged edge cracks. 


\section{Figure Captions}

Fig. 1 Configuration of the cracked strip.

Fig. 2 Normalized COD for zero fiber bridged edge crack with different relative orthotropy.

Fig. 3 Normalized crack mouth opening displacement vs. normalized loading parameter for bridged Griffith crack.

Fig. 4 Normalized total stress intensity factor vs. normalized loading parameter for bridged Griffith crack.

Fig. 5 Normalized shielding stress intensity factor vs. normalized loading parameter for bridged Griffith crack.

Fig. 6 Normalized crack mouth opening displacement vs. normalized loading parameter for bridged edge crack under uniform tension.

Fig. 7 Normalized total stress intensity factor vs. normalized loading parameter for bridged edge crack under uniform tension.

Fig. 8 Normalized shielding stress intensity factor v8. normalized loading parameter for bridged edge crack under uniform tension.

Fig. 9 Nondimensional parameters $\alpha, E / E_{m}$ and $E_{11} / E_{m}$ vs. fiber volume fraction $f$ for composite with $E_{f} / E_{m}=10, \nu_{f}=0.3$ and $\nu_{m}=0.35$.

Fig. 10 Nondimensional parameter $\alpha$ vs. fiber volume fraction $f$ for several composites with $\nu_{f}=0.3$ and $\nu_{m}=0.35$. 
Fig. 11 Nondimensional parameters $\alpha, E^{*} / E_{m}$ and $E_{11} / E_{m}$ vs. $E_{f} / E_{m}$ for composite with $\nu_{f}=0.3, \nu_{m}=0.35$ and $f=0.4$. 


\begin{tabular}{||c|c|c|c|c|c|c|c|}
\hline \multicolumn{8}{|c|}{$K / \sigma_{\infty} \sqrt{\pi a / 2}$} \\
\hline \multirow{2}{*}{$\lambda$} & $a / h$ & 0 & 1 & 2 & 3 & 4 & 5 \\
\cline { 3 - 8 } 0.2 & 0.1 & 1.247 & 1.190 & 1.167 & 1.154 & 1.146 & 1.140 \\
& 0.3 & 1.725 & 1.661 & 1.639 & 1.627 & 1.620 & 1.615 \\
& 0.5 & 2.964 & 2.833 & 2.783 & 2.755 & 2.738 & 2.726 \\
\hline & 0.1 & 1.247 & 1.190 & 1.167 & 1.154 & 1.146 & 1.140 \\
0.6 & 0.3 & 1.725 & 1.661 & 1.639 & 1.627 & 1.620 & 1.615 \\
& 0.5 & 2.964 & 2.833 & 2.783 & 2.755 & 2.738 & 2.726 \\
\hline & 0.1 & 1.247 & 1.190 & 1.167 & 1.154 & 1.146 & 1.140 \\
1.0 & 0.3 & 1.725 & 1.661 & 1.639 & 1.627 & 1.620 & 1.615 \\
& 0.5 & 2.964 & 2.833 & 2.783 & 2.755 & 2.738 & 2.726 \\
\hline
\end{tabular}

TABLE 1 NORMALIZED STRESS INTENSITY FACTOR VS. ORTHOTROPY RESCALING PARAMETERS $\lambda$ AND $\rho$ FOR ZERO FIBER BRIDGED EDGE CRACKS.

\begin{tabular}{|c|c|c|c|c|c|c|c|}
\hline \multicolumn{8}{|c|}{$[\boldsymbol{\sigma}] E^{\prime} / 2 \sigma_{\infty} h$} \\
\hline \multirow{2}{*}{$\lambda$} & $a / h$ & 0 & 1 & 2 & 3 & 4 & 5 \\
\cline { 2 - 8 } & 0.1 & 0.355 & 0.309 & 0.291 & 0.280 & 0.273 & 0.268 \\
0.2 & 0.3 & 1.575 & 1.406 & 1.342 & 1.308 & 1.286 & 1.271 \\
& 0.5 & 5.499 & 4.947 & 4.742 & 4.633 & 4.564 & 4.517 \\
\hline & 0.1 & 0.355 & 0.309 & 0.291 & 0.280 & 0.273 & 0.268 \\
0.6 & 0.3 & 1.575 & 1.406 & 1.342 & 1.308 & 1.286 & 1.271 \\
& 0.5 & 5.499 & 4.947 & 4.742 & 4.633 & 4.564 & 4.517 \\
\hline & 0.1 & 0.355 & 0.309 & 0.291 & 0.280 & 0.273 & 0.268 \\
1.0 & 0.3 & 1.575 & 1.406 & 1.342 & 1.308 & 1.286 & 1.271 \\
& 0.5 & 5.499 & 4.947 & 4.742 & 4.633 & 4.564 & 4.517 \\
\hline
\end{tabular}

TABLE 2 NORMALIZED CMOD VS. ORTHOTROPY RESCALING PARAMETERS $\lambda$ AND $\rho$ FOR ZERO FIBER BRIDGED EDGE CRACKS. 


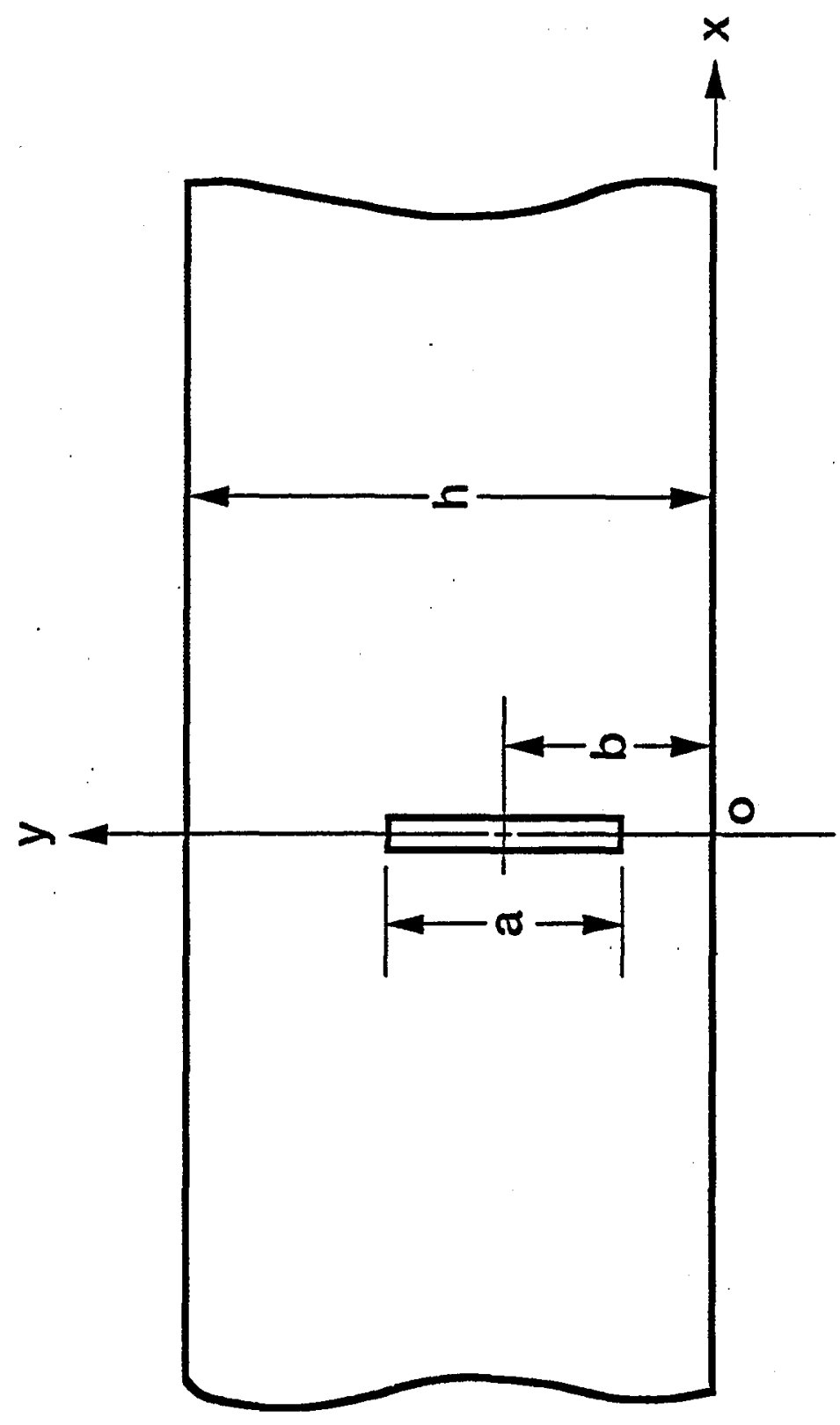

疍 


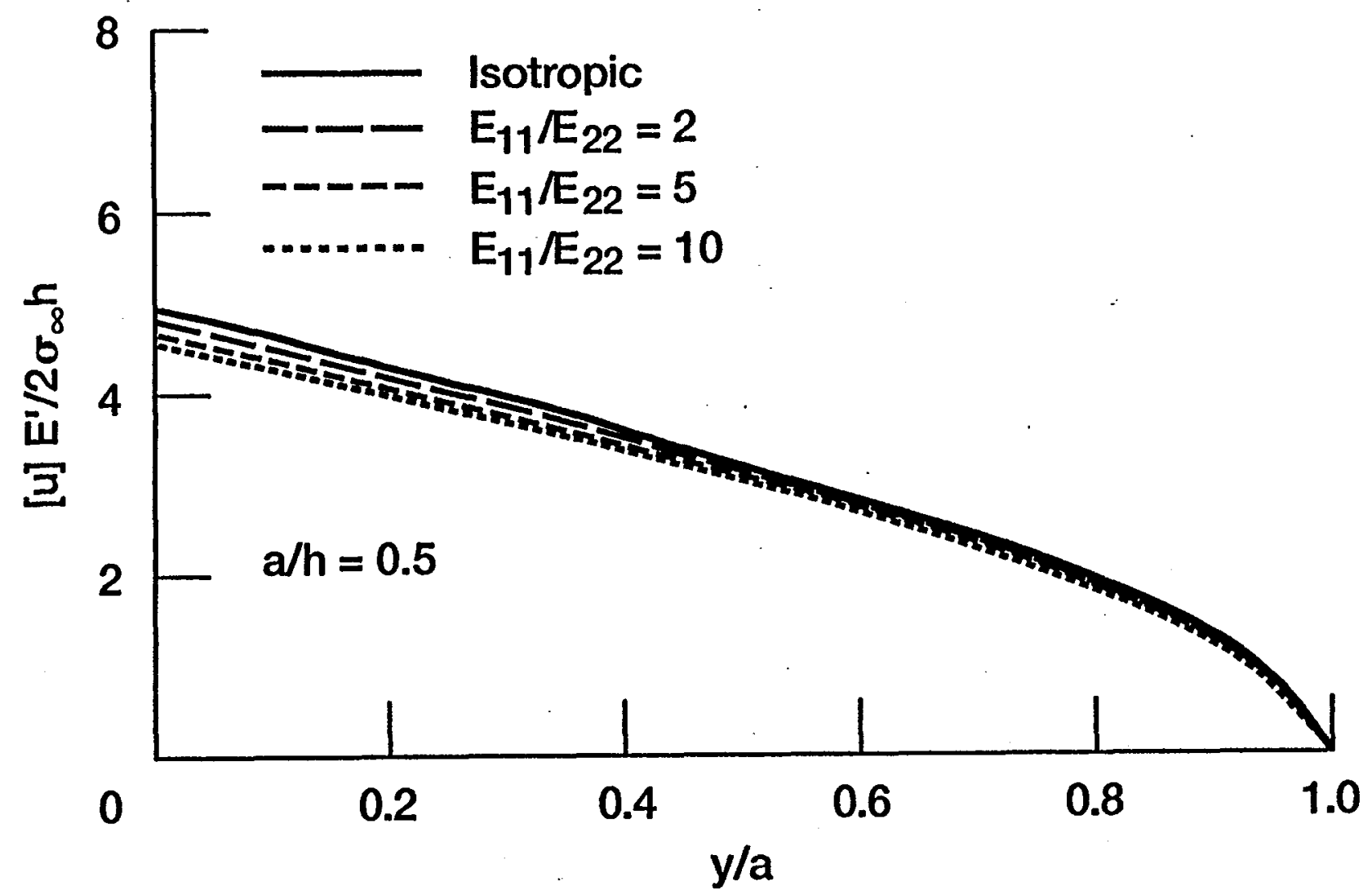

FIG. 2 NORMALIZED COD FOR ZERO FIBER BRIDGED EDGE CRACK WITH DIFFERENT RELATIVE ORTHOTROPY 


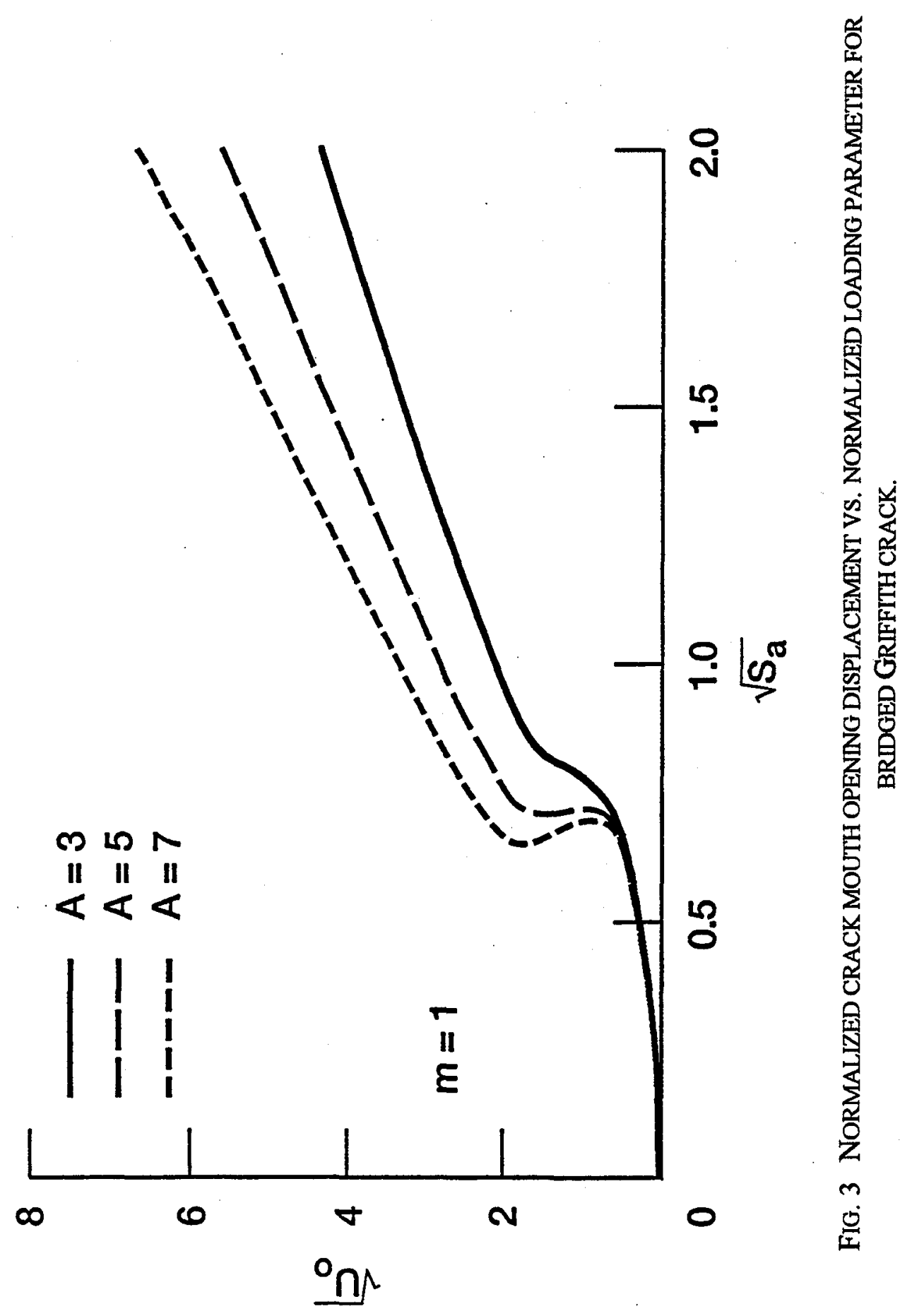




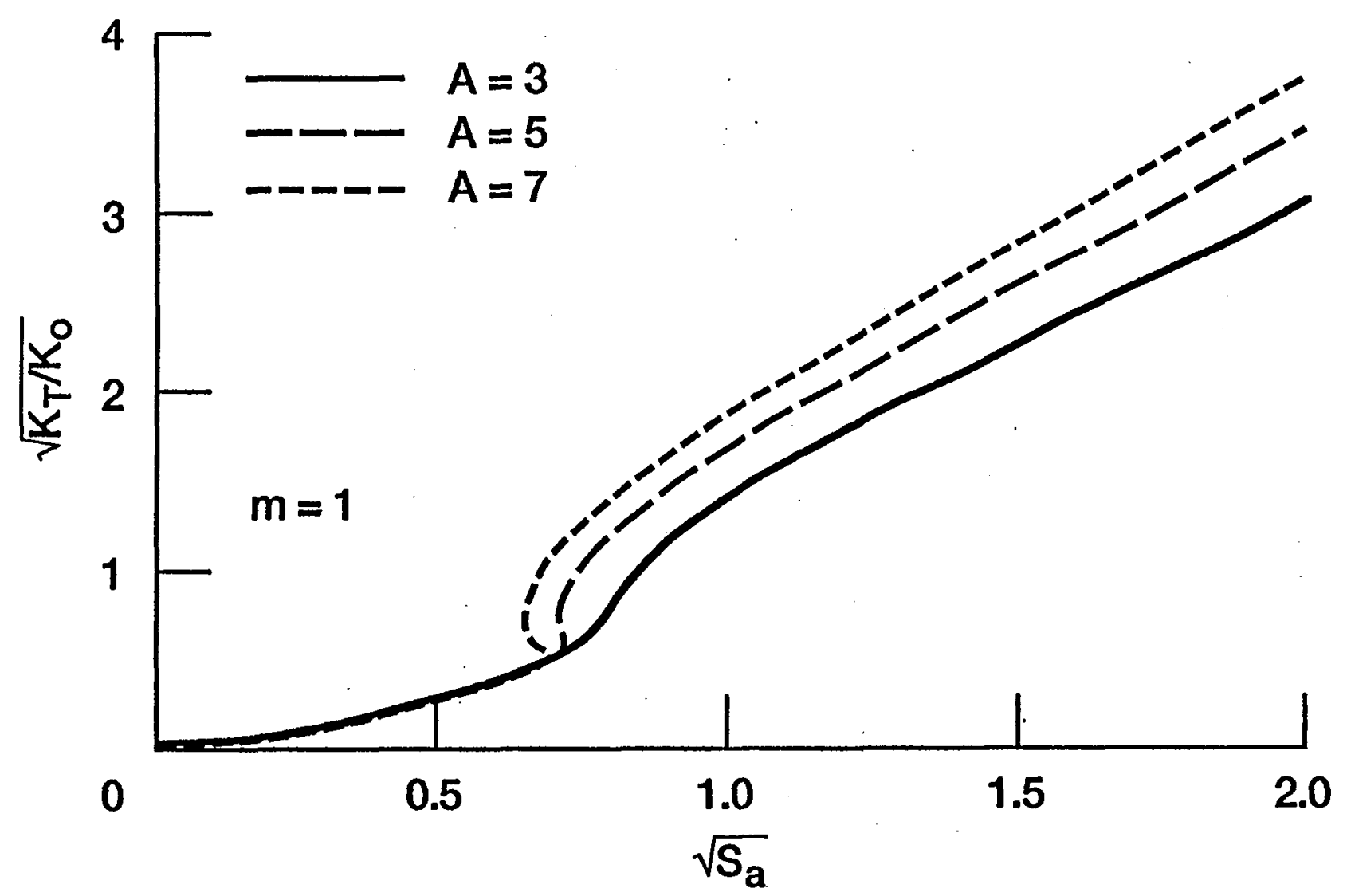

FIG. 4 NORMALIZED TOTAL STRESS INTENSITY FACTOR VS. NORMALIZED LOADING PARAMETER FOR BRIDGED GRIFFITH CRACK. 


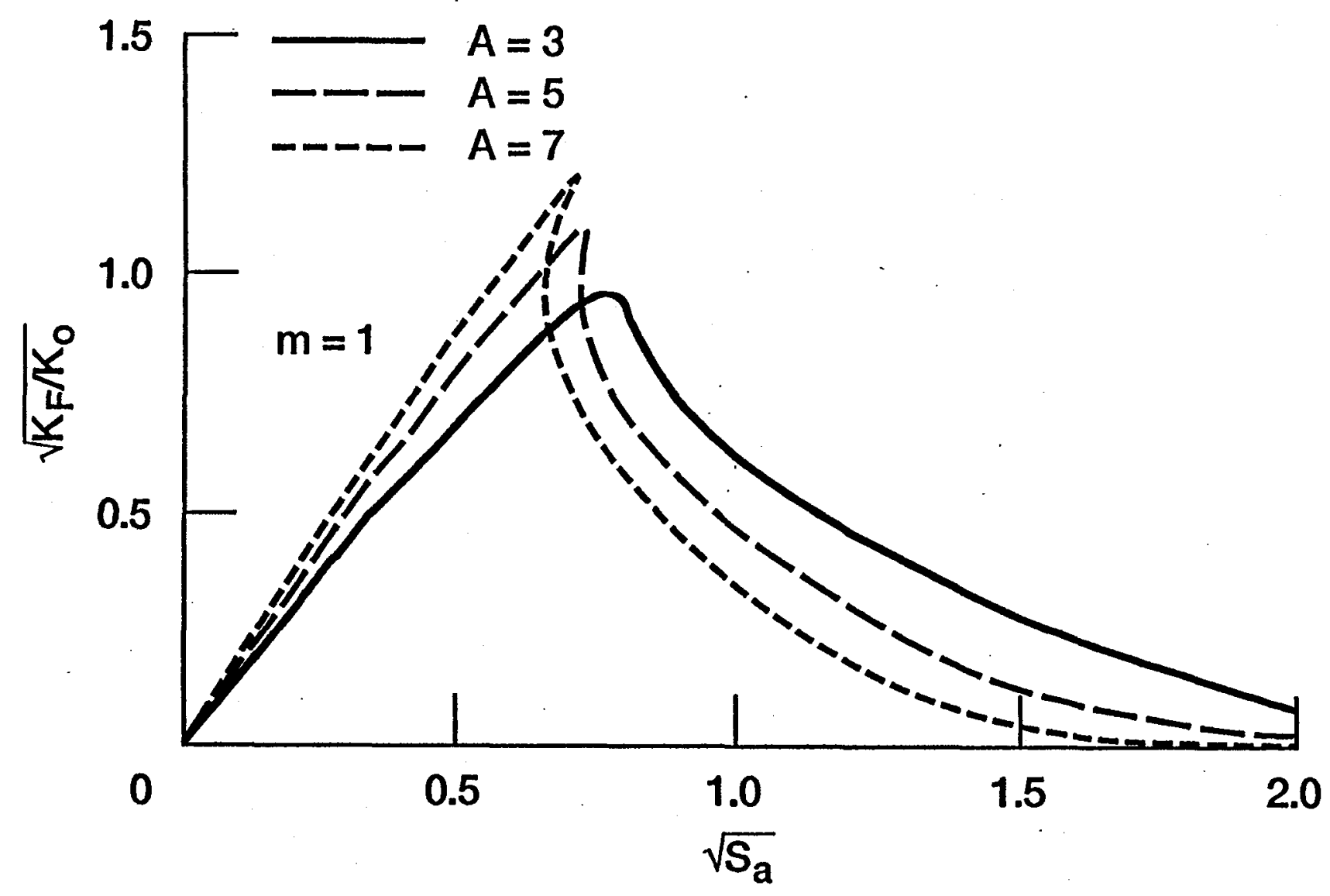

FIG. 5 NORMALIZED SHIELDING STRESS INTENSITY FACTOR VS. NORMALIZED LOADING PARAMETER FOR BRIDGED GRIFFITH CRACK. 


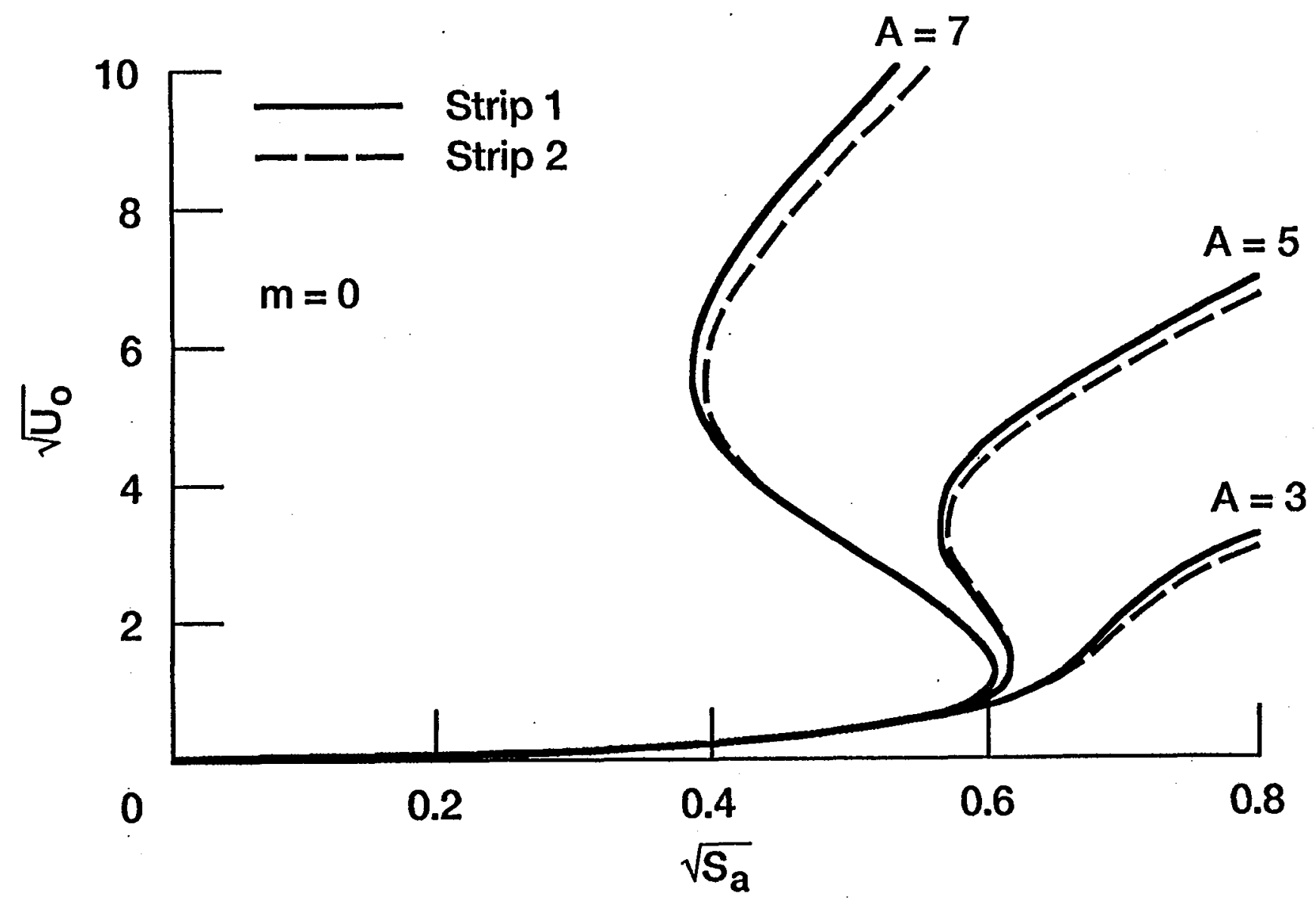

FIG. 6 NORMALIZED CRACK MOUTH OPENING DISPLACEMENT VS. NORMALIZED LOADING PARAMETER FOR BRIDGED EDGE CRACK UNDER UNIFORM TENSION. 


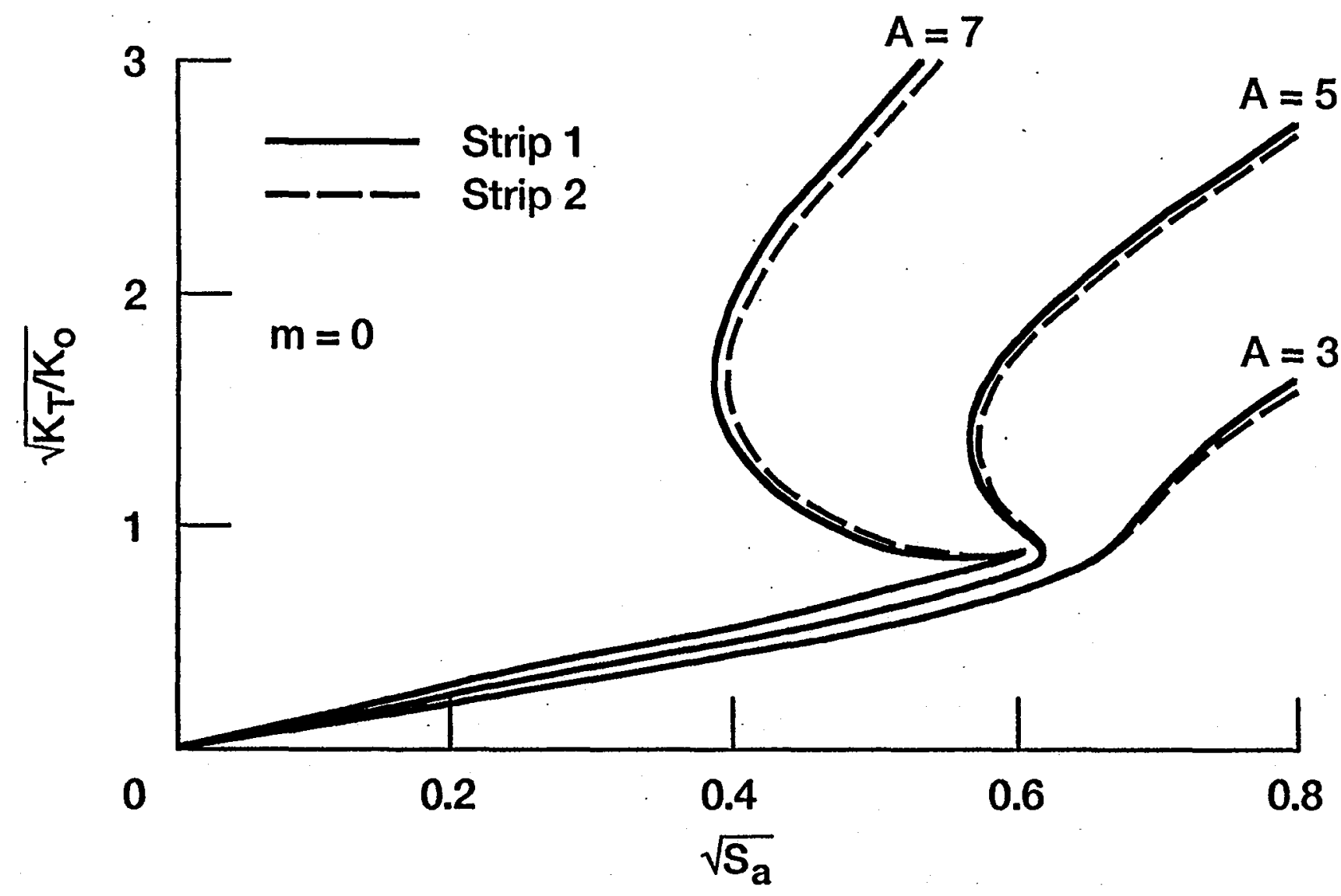

FIG. 7 NORMALIZED TOTAL STRESS INTENSITY FACTOR VS. NORMALIZED LOADING PARAMETER FOR BRIDGED EDGE CRACK UNDER UNIFORM TENSION. 


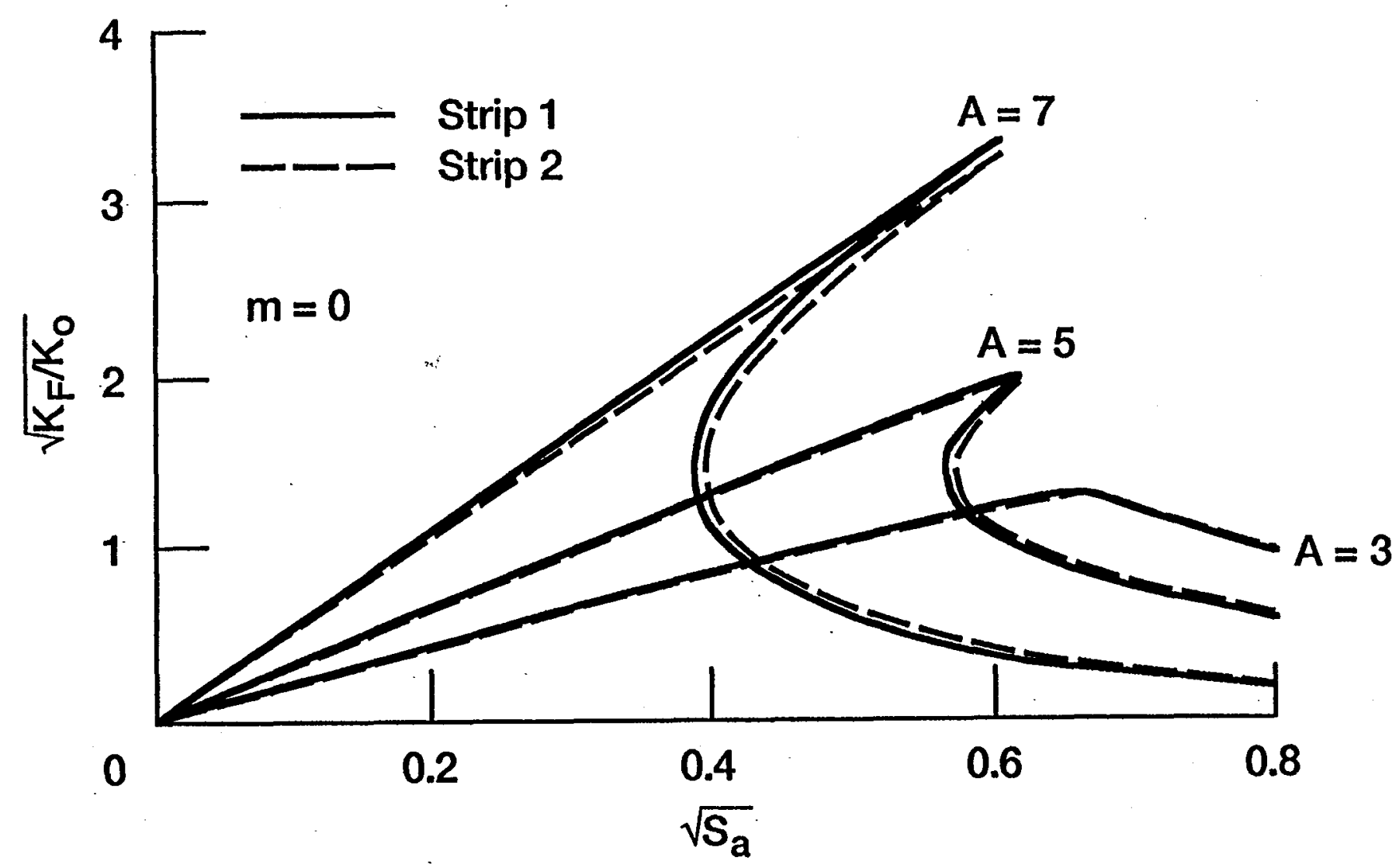

FIG. 8 NORMALIZED SHIELDING STRESS INTENSITY FACTOR VS. NORMALIZED LOADING PARAMETER FOR BRIDGED EDGE CRACK UNDER UNIFORM TENSION. 


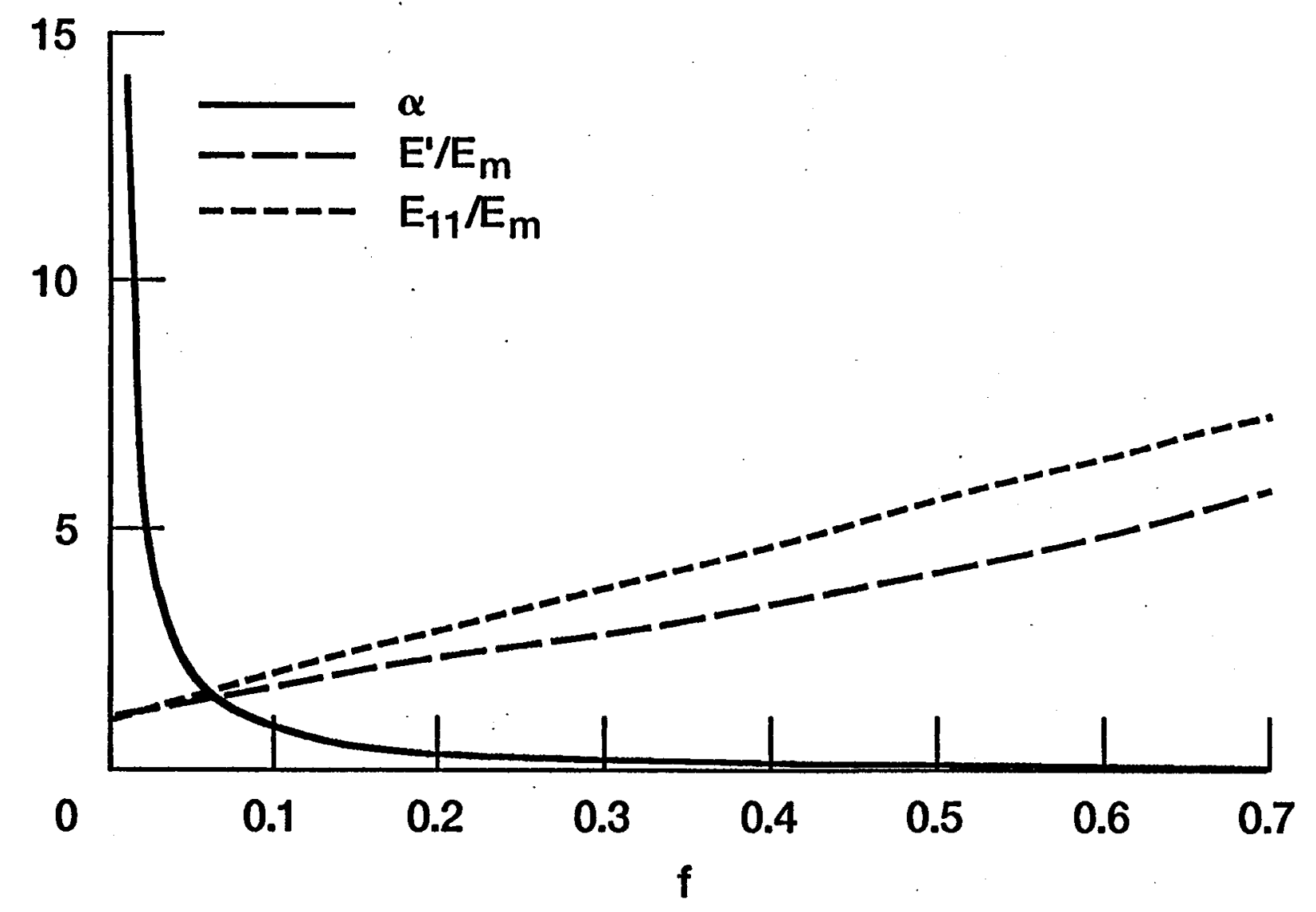

Fig. 9 NONDIMENSIONAL PARAMETERS $\alpha, E^{\prime} / E_{m}$ AND $E_{11} / E_{m}$ VS. FIBER VOLUME FRACTION $f$ FOR COMPOSITE WITH $E_{f} / E_{m}=10, v_{f}=0.3$ AND $v_{m}=0.35$. 


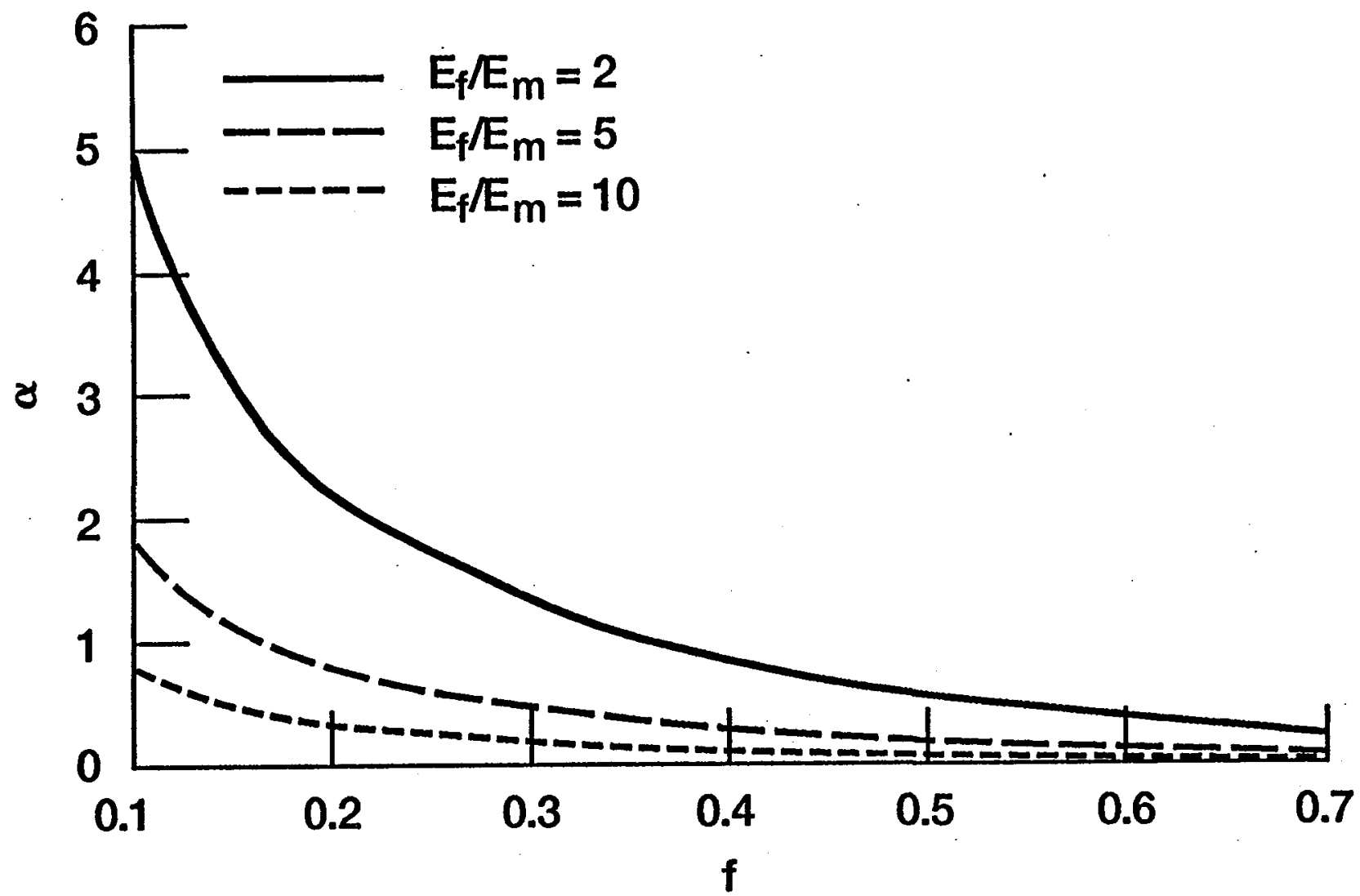

FIG. 10NONDIMENSIONAL PARAMETER $\alpha$ VS. FIBER VOLUME FRACTION $f$ FOR SEVERAL COMPOSITES WITH $v_{f}=0.3$ AND $v_{m}=0.35$. 


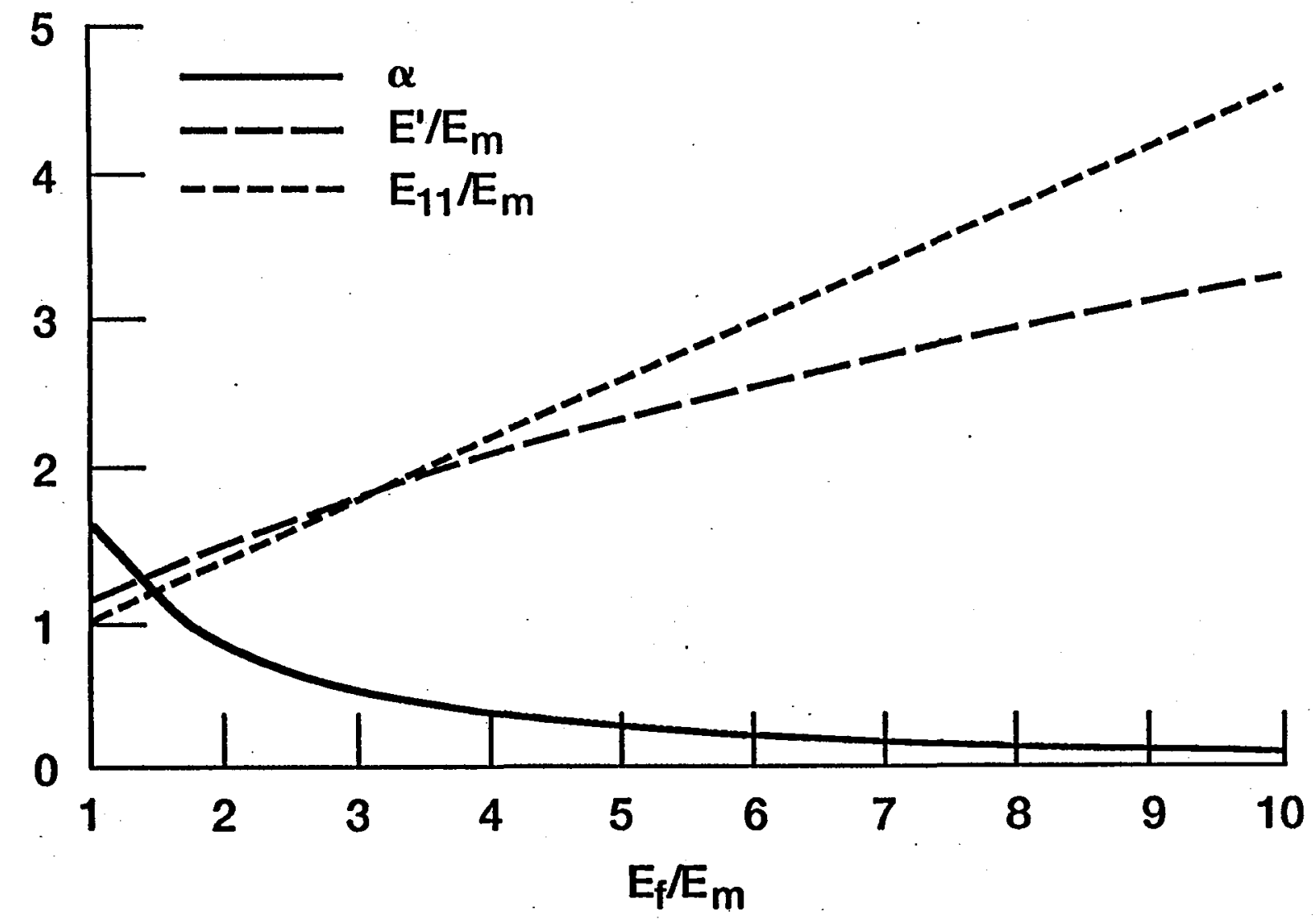

FIG. 11 NONDIMENSIONAL PARAMETERS $\alpha, E^{\prime} / E_{m}$ AND $E_{11} / E_{m}$ VS. $E_{f} / E_{m}$ FOR COMPOSITE WITH $v_{f}=0.3, v_{m}=0.35$ AND $f=0.4$. 
Public reporting burden for this collection of information is estimated to average 1 hour per response, including the time for reviewing instructions, searching existing data sources, gathering and maintaining the data needed, and completing and reviewing the collection of information. Send comments regarding this burden estimate or any other aspect of this collection of information, including suggestions for reducing this burden, to Washington Headquarters Services, Directorate for Information Operations and Reports, 1215 Jefte

\begin{tabular}{l|l|l} 
Davis Highway, Suite 1204, Arlington, VA 22202 & 3. REPORT TYPE AND DATES COVERED
\end{tabular}

\begin{tabular}{|l|c|c|}
\hline 1. AGENCY USE ONLY (Leave blank) & $\begin{array}{c}\text { 2. REPORT DATE } \\
\text { February } 1994\end{array}$ & $\begin{array}{c}\text { 3. REPOAT TYPE AND DATES COVERED } \\
\text { Final Contractor Report }\end{array}$
\end{tabular}

4. TITLE AND SUBTITLE

The Effects of Anisotropy on the Nonlinear Behavior of

Bridged Cracks in Long Strips

6. AUTHOR(S)

R. Ballarini and H.A. Luo
5. FUNDING NUMBERS

WU-505-63-5B

C-NAG3-856

\section{PERFORMING ORGANIZATION NAME(S) AND ADDRESS(ES)}

Case Western Reserve University

Department of Civil Engineering

Cleveland, Ohio 44106
8. PERFORMING ORGANIZATION REPORT NUMBER

$\mathrm{E}-8388$

9. SPONSORING/MONITORING AGENCY NAME(S) AND ADDRESS(ES)

10. SPONSORING/MONITORING AGENCY REPORT NUMBER

National Aeronautics and Space Administration

Lewis Research Center

Cleveland, Ohio 44135-3191

NASA CR-194453

11. SUPPLEMENTARY NOTES

R. Ballarini, Case Western Reserve University; and H.A. Luo, Shanghai Jiao Tong University, Department of Engineering Mechanics, Shanghai 200030, China. Project Manager, Noel N. Nemeth, Structures Division, organization code 5250, NASA Lewis Research Center, (216) 433-3215.

12a. DISTRIBUTIONAVAILABILITY STATEMENT

12b. DISTRIBUTION CODE

Unclassified - Unlimited

Subject Category 39

13. ABSTRACT (Maximum 200 words)

This paper presents a model which can be used to predict the two-dimensional nonlinear behavior of bridged cracks in orthotropic strips. The results obtained using a singular integral equation formulation which incorporates the anisotropy rigorously show that, although the effects of anisotropy are significant, the nondimensional quantities employed by Cox and Marshall (1991) can generate nearly universal results (R-curves, for example) for different levels of relative anisotropy. The role of composite constituent properties in the behavior of bridged cracks is clarified in this paper.

14. SUBJECT TERMS

Fracture mechanics; Ceramic matrix composites; Crack propagation 39

16. PRICE CODE

$\mathrm{A} 03$

17. SECURITY CLASSIFICATION OF REPORT

Unclassified
18. SECURITY CLASSIFICATION OF THIS PAGE

Unclassified
19. SECURITY CLASSIFICATION OF ABSTRACT Unclassified 